\title{
Searching for X-ray counterparts of Fermi Gamma-ray pulsars in Suzaku observations
}

\author{
Yu Aoki ${ }^{1}$, Takahiro Enomoto ${ }^{1}$, Yoichi Yatsu ${ }^{1}$, Nobuyuki Kawai ${ }^{1}$, \\ Takeshi Nakamori ${ }^{2}$, Jun Kataoka ${ }^{2}$ and P. Saz Parkinson ${ }^{3}$ \\ ${ }^{1}$ Dept. of Physics, Tokyo Institute of Technology, \\ B2-12-1 Oookayama Meguro Tokyo, 152-8551, Japan \\ email: aoki@hp.phys.titech.ac.jp \\ ${ }^{2}$ Research Institute for Science and Engineering, Waseda University, Japan \\ ${ }^{3}$ Santa Cruz Institute for Particle Physics, Dept. of Physics and Dept. of Astronomy and \\ Astrophysics, University of California at Santa Cruz, Santa Cruz, CA 95064, USA
}

\begin{abstract}
We report the Suzaku follow-up observations of the Gamma-ray pulsars, 1FGL J0614,13328, J1044.55737, J1741.82101, and J1813.31246, which were discovered by the Fermi Gamma-ray observatory. Analysing Suzaku/XIS data, we detected X-ray counterparts of these pulsars in the Fermi error circle and interpreted their spectra with absorbed power-law functions. These results indicate that the origin of these X-ray sources is non-thermal emission from the pulsars or from Pulsar Wind Nebulae (PWNe) surrounding them. Moreover we found that J1741.82101 exhibits a peculiar profile: spin-down luminosity vs flux ratio between X- and gamma-rays is unusually large compared to usual radio pulsars.
\end{abstract}

Keywords. Pulsar, Pulsar wind nebulae, Gamma-ray pulsars. Fermi.

\section{Introduction}

The Fermi gamma-ray observatory has discovered more than three thousands of gamma-ray sources. About 10 percent of the newly found objects were categorized into pulsars and pulsar wind nebulae. We focused on the newly found gamma-ray pulsars to study their nature. For this purpose we observed four bright gamma-ray pulsars with the Suzaku X-ray observatory. Here we present a summary of the observations.

\section{Observation and analysis}

Suzaku is the fifth Japanese X-ray observatory with 4 X-ray telescopes. Thanks to the low-earth orbit the particle background is low compared with the other large X-ray observatories. So it is suited for searching diffuse emissions in the universe. We conducted observations on 2010. Each exposure time was $20 \mathrm{ks}$.

First we performed astrometry. From the obtained X-ray images we discovered point sources clearly. These locations are consistent with the Fermi's error circles. The position of these point sources are also consistent with the timing position determined by radio follow-up observations. These positional coincidences strongly support that the discovered X-ray sources are the X-ray counterparts of gamma-ray pulsars.

Next we studied the spectroscopy. In order to accumulate source photons we chose circle regions with a radius of 1 arcmin centered on the pulsars. The obtained X-ray spectra were well modelled by absorbed power-law functions. The results of spectral fitting are summarized in Table. 1. 1FGL J1813,3-1246 shows a very flat spectrum with a photon index of 0.8 that cannot be explained by the standard acceleration model. While the other pulsars show relatively flat spectra. 
Table 1. Fit results with power-law model or interstellar absorbed power-law model for the spectra of four pulsars

\begin{tabular}{c|ccc|c}
\hline name & $\begin{array}{c}n_{H} \\
{\left[\times 10^{22} \mathrm{~cm}^{2}\right]}\end{array}$ & Index & $\begin{array}{c}\text { Flux }(0.5-10.0 \mathrm{keV}) \\
{\left[\mathrm{erg} / \mathrm{s} / \mathrm{cm}^{2}\right]}\end{array}$ & $\chi^{2} /$ D.O.F \\
\hline J0614.1-3328 & - & $2.63_{-0.27}^{+0.30}$ & $5.73 \pm 0.67 \times 10^{-14}$ & $11.95 / 14$ \\
$\mathrm{~J} 1044.5-5737$ & - & $2.00_{-0.34}^{+0.36}$ & $7.50_{-1.12}^{+1.22} \times 10^{-14}$ & $5.56 / 8$ \\
$\mathrm{~J} 1741.8-2101$ & $0.28_{-0.09}^{+0.11}$ & $2.81_{-0.20}^{+0.23}$ & $6.56_{-0.97}^{+1.35} \times 10^{-13}$ & $7.44 / 24$ \\
$\mathrm{~J} 1813.3-1246$ & $1.36_{-0.27}^{+0.31}$ & $0.79 \pm 0.16$ & $1.27 \pm 0.06 \times 10^{-12}$ & $9.25 / 20$ \\
\hline
\end{tabular}

\section{Discussion}

We estimate the X-ray luminosity from the X-ray flux for each pulsar using equation (1) which is the empirical formula between X-ray luminosity and spindown luminosity (Kanai D-thesis).

$$
\frac{L_{X}}{10^{32} \mathrm{erg} / \mathrm{s}}=(2.99 \pm 1.08) \times\left(\frac{L_{s d}}{10^{36} \mathrm{erg} / \mathrm{s}}\right)^{1.10 \pm 0.14}
$$

Then we calculated the distance and Gamma-ray luminosity of the four pulsars assuming $L_{X, \Gamma}=4 \pi d^{2} f F_{X, \Gamma}$ from obtained X-ray flux, X-ray luminosity, and Gamma-ray flux.

Next, we compared the four pulsars to other Fermi pulsars used in Kanai D-thesis. Fig. 1 and 2 show the spin-down luminosity vs. Gamma-ray luminosity and spin-down luminosity vs. the $\mathrm{X} /$ Gamma flux ratio, respectively.

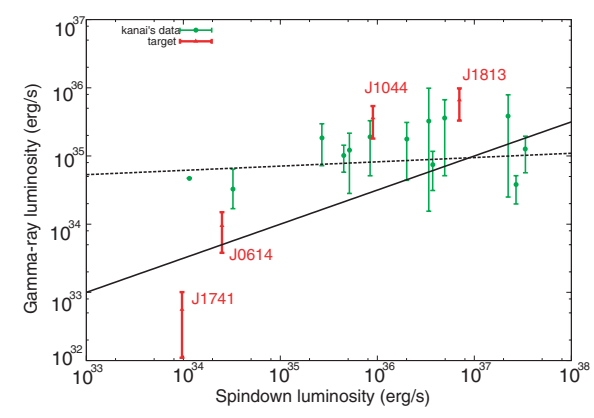

Figure 1. Relation between spindown luminosity and Gamma-ray luminosity for four pulsars and other Fermi pulsars

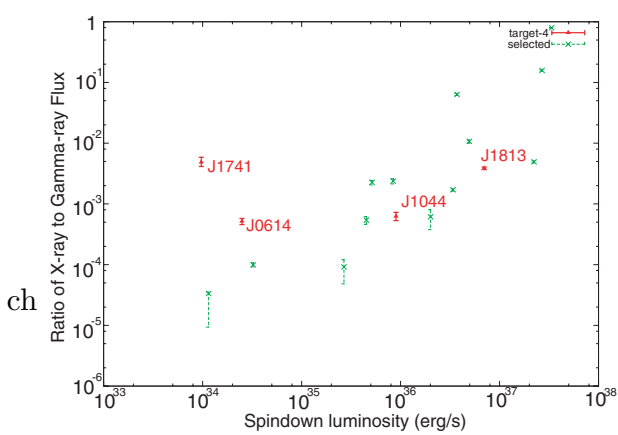

Figure 2. Relation between spindown luminosity and the ratio of X-ray and Gamma-ray flux for four pulsars and other Fermi pulsars

\section{References}

Amari, S., Hoppe, P., Zinner, E., \& Lewis R. S. 1995, Meteoritics, 30, 490

Anders, E. \& Zinner, E. 1993, Meteoritics, 28, 490

Bernatowicz, T. J., Messenger, S., Pravdivtseva, O., Swan, P., \& Walker, R. M. 2003, Geochim. Cosmochim. Acta, 67, 4679

Busso, M., Gallino, R., \& Wasserburg, G. J. 1999, ARAA, 37, 239

Croat, T. K., Stadermann, F. J., \& Bernatowicz, T. J. 2005, ApJ, 631, 976

Draine, B. T. 2003, ARAA, 41, 241

Hoppe, P. \& Zinner, E. 2000, J. Geophys. Res., A105, 10371

Hoppe, P., Ott, U., \& Lugmair, G. W. 2004, New Astron. Revs, 48, 171

Kanai.Y., 2010, Ph.D. thesis, Tokyo Institute of Technology

Lodders, K. \& Fegley, B. 1998, Meteorit. Planet. Sci., 33, 871

Meyer, B. S., Clayton, D. D., \& The, L.-S. 2000, ApJ (Letters), 540, L49 